$\mathrm{DE}$

M E D I C I N A

T R O P I C A L

$\mathrm{DE}$

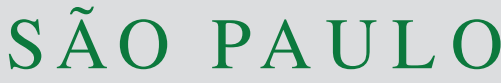

JOURNAL OF THE SÃO PAULO INSTITUTE OF TROPICAL MEDICINE

1Universidade de São Paulo, Hospital das Clínicas, Instituto da Criança, Unidade de Terapia Intensiva Pediátrica, São Paulo, São Paulo, Brazil

2Universidade de São Paulo, Hospital das Clínicas, Instituto da Criança, Departamento de Infectologia Pediátrica, São Paulo, São Paulo, Brazil

${ }^{3}$ Universidade de São Paulo, Hospital das Clínicas, Instituto da Criança, Departamento de Reumatologia Pediátrica, São Paulo, São Paulo, Brazil

${ }^{4}$ Universidade de São Paulo, Hospital das Clínicas, Departamento de Patologia, São Paulo, São Paulo, Brazil

Correspondence to: Amaro Nunes Duarte-Neto

Universidade de São Paulo, Hospital das Clínicas, Departamento de Patologia, Avenida Dr. Arnaldo, 455, Cerqueira Cesar, CEP 01246-903 São Paulo, SP, Brazil Tel: +55 11 98546-4225

E-mail: amaro.ndneto@ hc.fm.usp.br

Received: 27 March 2019

Accepted: 15 July 2019

\section{Acute petrified myocardium associated with meningococcal sepsis in childhood-onset systemic lupus erythematous: a fatal case}

\author{
Ana Carolina Etrusco Zaroni Santos ${ }^{\circledR 1}$, Michele Luglio ${ }^{\circledR 1}$, Artur Figueiredo \\ Delgado ${ }^{(1)}$, Constance Dell Santo Vieira Schuwartz ${ }^{\mathbb{1} 2}$, Heloísa Helena \\ Sousa Marques ${ }^{\circledR 2}$, Clarissa Carvalho de Miranda Valões ${ }^{(1)}$, Maria Andreina \\ Cabrera Dominguez ${ }^{(1,}$, Werther Brunow de Carvalho ${ }^{(1)}$, Clovis Artur \\ Silva ${ }^{1}$, Amaro Nunes Duarte-Neto ${ }^{(1)}$
}

\section{ABSTRACT}

Acute petrified myocardium associated with septic shock, diagnosed by autopsy has rarely been described. A 15-year-old adolescent male was diagnosed with childhood-onset systemic lupus erythematosus. One year later, he was hospitalized with fever, myalgia, headache, arthritis, vomiting, dyspnea and was diagnosed with sepsis secondary to bronchopneumonia and meningitis. Blood culture identified Neisseria meningitidis serogroup Y. Despite antibiotics and intensive therapeutic measures, he died after 29 days of hospitalization. The autopsy revealed necrotic cardiomyocytes with dystrophic calcification and interstitial fibrosis.

KEYWORDS: Myocardial calcification. Sepsis. Neisseria meningitides. Lupus. Intensive care.

\section{INTRODUCTION}

Systemic lupus erythematosus (SLE) is a chronic inflammatory autoimmune disease with concomitant or additive involvement of various organs and systems. The childhood-onset systemic lupus erythematosus (cSLE) is more severe than adult SLE, with higher morbidity and mortality rates ${ }^{1}$. Severe sepsis associated with cardiac dysfunction is a major predictor of poor prognosis in cSLE patients, particularly sepsis due to Staphylococcus aureus and Pseudomonas aeruginosa infections ${ }^{2}$.

The relationship between sepsis and myocardial calcification, also called "acute petrified myocardium", "petrified heart of sepsis" or "heart of stone", has been described in few animal models and case reports ${ }^{3-5}$. To our knowledge, no case report of petrified heart in cSLE currently exists. Therefore, we report herein a case of an adolescent male with cSLE who developed fatal meningococcal sepsis associated with severe acute petrified myocardium observed at a postmortem examination. The Ethical Committee of our University Hospital approved this study (Process No CAAE 09231912.2.1001.0068).

\section{CASE REPORT}

An adolescent male aged 15 years and three months was diagnosed in another hospital with cSLE based on the presence of arthritis, proteinuria (1.2 g/day), 
lymphopenia, thrombocytopenia and 1:640 positivity of antinuclear antibodies (speckled pattern), as well as anti-double stranded DNA antibodies (ELISA, > $200 \mathrm{IU} / \mathrm{mL}$ [positive $\geq 20 \mathrm{IU} / \mathrm{mL}$ ]) and low complement levels (C3- $43 \mathrm{mg} / \mathrm{dL}$ [normal range $90-180 \mathrm{mg} / \mathrm{dL}$ ]), C4- $4.5 \mathrm{mg} / \mathrm{dL}$ [normal range $10-40 \mathrm{mg} / \mathrm{dL}$ ]).

Routine non live immunizations (tetanus, hepatitis B, seasonal influenza, pneumococcus and Haemophilus) had been previously administered. At 16 years and three months, he was hospitalized at our University Hospital with fever, myalgia, arthritis, vomiting, dyspnea and clinical signs of sepsis. There were no meningeal signs. On the same day, he developed a severe and acute headache, followed by generalized tonic-clonic seizures that required orotracheal intubation. The chest X-ray showed perihilar infiltrate on the right side. Laboratory exams revealed: hemoglobin $13.1 \mathrm{~g} / \mathrm{dL}$ (normal range 14-18 g/dL), hematocrit 37\%, white blood cell count $10,100 / \mathrm{mm}^{3}(1 \%$ metamielocytes, $15 \%$ bands, $68 \%$ segmented neutrophils, $6 \%$ lymphocytes and $10 \%$ monocyte), platelets $70,000 / \mathrm{mm}^{3}$, proteinuria (3.2 g/24 h), urinalysis (leukocytes 6,000 high-power field and erythrocytes 432,000 high-power field), urea $56 \mathrm{mg} / \mathrm{dL}$ (normal range 10-42 mg/dL), creatinine $3.59 \mathrm{mg} / \mathrm{dL}$ (normal range $0.5-0.9 \mathrm{mg} / \mathrm{dL}$ ), C3- $25 \mathrm{mg} / \mathrm{dL}$ (normal range $90-180 \mathrm{mg} / \mathrm{dL}$ ), C4- $2.1 \mathrm{mg} / \mathrm{dL}$ (normal range $10-40 \mathrm{mg} / \mathrm{dL}$ ), and C-reactive protein $368 \mathrm{mg} / \mathrm{dL}$ (normal range $<5 \mathrm{mg} / \mathrm{dL}$ ). No abnormalities were observed on the cranial tomography and the echocardiography. A lumbar puncture was performed, and the cerebrospinal fluid (CSF) analysis showed the following: white cell count $36 / \mathrm{mm}^{3}$ (88\% neutrophils, $8 \%$ lymphocytes), glucose $16 \mathrm{mg} / \mathrm{dL}$ (normal range $40-80 \mathrm{mg} / \mathrm{dL}$ ) and protein $456 \mathrm{mg} / \mathrm{dL}$ (normal range $15-40 \mathrm{mg} / \mathrm{dL}$ ). The initial clinical diagnoses were bronchopneumonia and meningitis, and the treatment included intravenous hydration, antibiotics (vancomycin, amikacin, meropenem and micafungin), vasoactive and inotropic drugs (norepinephrine, dobutamine and milrinone), mechanical ventilation and continuous renal replacement therapy.

Immunological tests revealed positive results for antidsDNA antibodies and $\operatorname{IgG}(58.79 \mathrm{GPL} / \mathrm{mL})$ and $\operatorname{IgM}$ (31.45 MPL/mL) anticardiolipin antibodies. On the third day of hospitalization, peripheral blood culture identified Neisseria meningitidis serogroup Y with intermediate sensitivity to penicillin. The CSF culture was negative. Ten days after admission, the SLEDAI- $2 \mathrm{~K}^{6}$ was 21 and pulse therapy was administered without meaningful response. His oxygenation index and ventricular function progressively worsened. At this point, a second echocardiograph showed left ventricle ejection fraction of $49 \%$ (normal $\geq 55 \%$, by the Teichholz method) and mild enlargement of the left ventricle and both atrium chambers. Despite all therapeutic and supportive measures, he died after 29 days of hospitalization. Serology tests for hepatitis (A, $\mathrm{B}$ and C), HIV, Epstein Barr virus, cytomegalovirus and toxoplasmosis were all negative.

The autopsy was performed after written consent was obtained from his next of kin. It revealed bilateral and extensive pneumonia, mild pleuritis and pericarditis, active diffuse proliferative nephritis and foci of chronic cerebral abscess in the left temporal cortical area. Remarkably, the heart was heavy, weighting $532 \mathrm{~g}$ (reference range 200-350 g) and stiff, and the cut surface had goldenyellowish rough areas scattered in both ventricles (Figure 1A-1C). Under microscopy, these yellowish and rough areas were necrotic cardiomyocytes with dystrophic calcification in their cytoplasm that were associated with interstitial myocardial edema and fibrosis, without myocardial vasculitis (Figure 1D, 1E, 1G, 1H). Von Kossa staining highlighted the calcifications in black (Figure 1F). The pericardium, coronary vessels and atrioventricular valves were preserved.

Myocardial analysis by electron microscopy showed severe damage to cardiomyocyte fibers and mitochondria, with intense myocytolysis (Figure 1J), edema, cristolysis and calcium accumulation in mitochondria (Figure 1M). In more intense lesions, calcification takes over the cellular content (Figure 1K, 1L). Interstitial and endothelial edema were other findings.

\section{DISCUSSION}

We reported the first case of acute petrified myocardium most likely induced by a meningococcal sepsis in an active cSLE patient.

Infections are a relevant cause of morbidity and mortality in cSLE patients. The most important risk factors associated with severe sepsis in cSLE populations are related to the disease itself (disease duration, lymphopenia, disease activity and hypocomplementemia) and treatment (glucocorticoid), as observed in our patient. Primary immunodeficiencies (particularly $\mathrm{C} 2, \mathrm{C} 4, \mathrm{C} 1 \mathrm{q}$ and $\mathrm{C} 1 \mathrm{r}-\mathrm{s}$ deficiencies) could also contribute to severe infection in our patient; however, these abnormalities were not assessed during hospitalization ${ }^{2}$.

Of note, sepsis was caused by a meningococcal disease (serogroup Y) in the present cSLE patient. In Brazil, this infection in children and young adults is predominantly caused by serogroups C (71\%), B (19\%) and W (6\%), and rarely caused by serogroup $\mathrm{Y}^{7}$. Our patient had not been immunized with the anti-meningococcal vaccine (MenACWY conjugate vaccine) as the introduction of this 

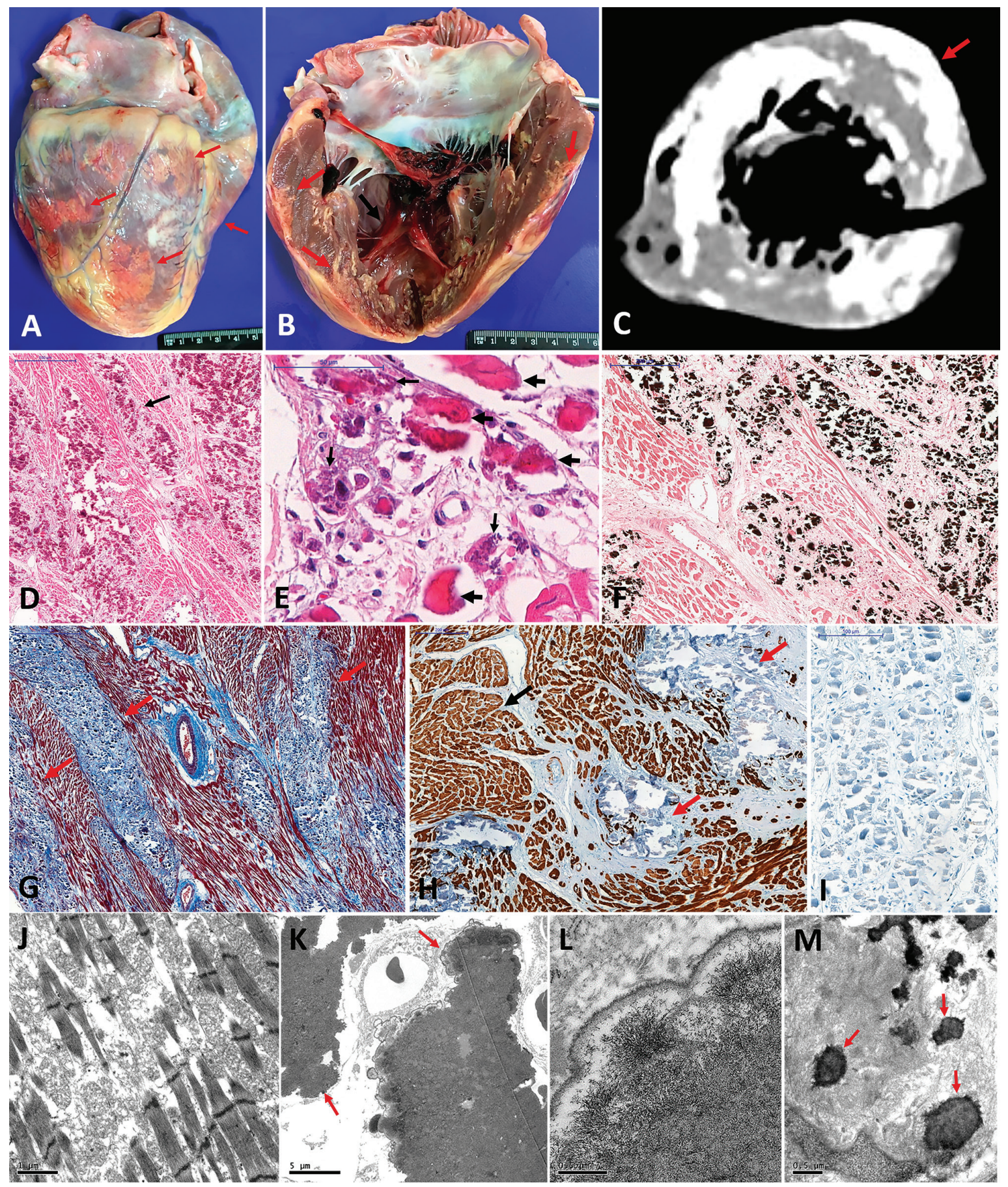

Figure 1 - Pathological findings in a heart with acute petrified myocardium: A) Gross exam of the heart (dorsal view) shows scattered yellowish subepicardial calcified plaques (arrow); B) scattered yellowish and calcified plaques (red arrows) in the myocardium on the left ventricle. An organized thrombus inside the cardiac left chamber (black arrow); C) postmortem, ex situ computerized tomography scan of the heart shows diffuse and coalescent hyperdense areas (calcification) in the entire myocardium on the ventricles, mainly in the left ventricle (arrow); D) necrotic cardiomyocytes with eosinophilic calcification and interstitial fibrosis (H\&E); E) the calcification is within the cardiomyocyte cytoplasm and has lamellar (thick arrow) or granular (thin arrow) aspect (H\&E); F) calcified cardiac fibers labeled in black with Von Kossa stain; G) interstitial collagen deposition (arrows) in the myocardium in the foci of necrotic and calcified cardiomyocytes (Masson trichrome stain); H) loss of desmin expression in necrotic and calcified cardiomyocytes (red arrows). Non-necrotic fibers maintain desmin expression and are labeled in brown (black arrow) (immunohistochemistry, anti-desmin, mouse monoclonal antibody, Cell Marque ${ }^{\mathrm{TM}}$ ); I) negative expression of Bcl2 in necrotic cardiomyocytes (immunohistochemistry, anti-Bcl2, mouse monoclonal antibody, Cell Marque ${ }^{\mathrm{TM}}$ ); J-M) electron microscopy analysis of thin sections of the myocardium shows diffuse and intense myocytolysis and mitochondrial crystolisis and edema (in J), calcification confined to cardiomyocyte cytoplasm (arrows in $\mathrm{K}$ ), spicules of calcium with cottony aspect at the periphery of dense and lamellar calcification (in L), calcification in the entire mitochondrial matrix (arrows in M). Scale bars: $L, M=0.5 \mu \mathrm{m} ; \mathrm{J}=1 \mu \mathrm{m} ; \mathrm{K}=5 \mu \mathrm{m} ; \mathrm{E}=50 \mu \mathrm{m} ; \mathrm{I}=100 \mu \mathrm{m} ; \mathrm{F}, \mathrm{H}=200 \mu \mathrm{m} ; \mathrm{D}, \mathrm{G}=500 \mu \mathrm{m}$. 
vaccine was recent and it is not available in Brazilian public health care services. Thus, the presence of uncommon meningococcal serogroups not covered by the national vaccination program should be considered, and whenever possible, all prophylactic measures available should be implemented, especially in patients with risk factors.

Regarding the response to antibiotic treatment, in Brazil, there is no record of meningococcus resistance to penicillin, but $49 \%$ have an intermediate sensitivity profile ${ }^{7}$. The prevalence of penicillin resistance in serogroup $\mathrm{Y}$ is higher than that in the other groups. In the present case, the isolated meningococcal strain $Y$ had intermediate resistance to penicillin.

Acute diffuse myocardial calcification is a rare sepsisrelated phenomenon and has not been described in cSLE patients. Few cases associated with severe sepsis have been reported in the literature, and the majority of them had myocardial calcification in patients with neoplasia or severe hematological abnormalities ${ }^{4,5}$.

There are two mechanisms that describe calcium deposition in cardiac myocytes: metastatic calcification and dystrophic calcification. Metastatic calcification is characterized by disturbances in calcium and phosphorus metabolism, leading to high serum calcium and phosphorus levels and deposition of calcium in previously normal tissue. Dystrophic calcification is the most common and occurs when there is a deposition of calcium in degenerated or dead cardiac myocytes ${ }^{8}$. This is usually associated with infarction and severe sepsis. Cell membrane damage leads to increased permeability, resulting in an uncontrolled influx of calcium ions. In sepsis, cells can be damaged by ischemia and increased levels of endogenous and exogenous catecholamine, as well as factors reducing membrane protection $^{8}$.

A single or repeated excess of catecholamine is known to cause disseminated necrosis of cardiac myocytes ${ }^{9}$. In the present case, the patient used norepinephrine at low doses (up to $0.3 \mathrm{mcg} / \mathrm{kg} / \mathrm{min}$ ) for several days. However, he received dobutamine and milrinone for an extended time ( $\geq$ seven days).

Experimental animal models identified genes associated with myocardial protective effect on sepsis. Among them, $b c l-2$, with anti-apoptotic action, stands out as an inducer of mitochondrial resistance to injury caused by the intracellular calcium influx. In the present case, we performed immunohistochemistry to detect desmin and bcl-2 antigens expression in the myocardium, with negative expression in areas with cardiomyocyte necrosis and dystrophic calcification (Figure 1H, 1I), suggesting a lack of involvement of bcl-2 protein in acute petrified myocardial associated with sepsis ${ }^{10}$.
Proteins that influence cardiac contractility, such as UCP 2 (uncoupling protein 2), which has a protective myocardial effect, reduce mitochondrial edema and free radical release, and PARP (poly adenosine 5'-diphosphateribose polymerase), an enzyme responsible for accelerating the oxidative stress and the heart damage, has also been associated with the pathophysiology of myocardial injury in sepsis. Immunohistochemistry to detect the expression of these proteins in the cardiac muscle was not performed in the present case $\mathrm{s}^{11,12}$.

In our patient, the primary mechanism is likely to be dystrophic calcification due to septic shock, with calcium overload being the crucial pathogenic factor. Other factors, such as ischemia, acute kidney injury with renal replacement therapy, gene expression profile and vasoactive drug use, may have contributed to cell damage. The absence of myocardial vasculitis and other cardiac involvement associated with SLE in the autopsy suggests that the disease activity may be irrelevant to this acute myocardium calcification process.

Currently, there are no treatment for restrictive cardiomyopathy of acute petrified myocardium. Broad antibiotic therapy and inotropic and vasoactive drugs do not seem to change the fatal outcome, as was evident in this case. Prompt heart transplantation seems to be the only available treatment for patients in this condition ${ }^{5}$.

In conclusion, this is a rare case of acute restrictive cardiomyopathy with petrified myocardium associated with meningococcal sepsis in a cSLE patient with active disease.

\section{CONFLICT OF INTERESTS}

The authors declare no conflict of interests.

\section{FINANCIAL SUPPORT}

This study was supported by grants from Fundação de Amparo à Pesquisa do Estado de São Paulo (FAPESP 2015/03756-4 to CAS), Conselho Nacional de Desenvolvimento Científico e Tecnológico (CNPq 303422/2015-7 to CAS), Federico Foundation (to CAS) and by Núcleo de Apoio à Pesquisa "Saúde da Criança e do Adolescente" da USP (NAP-CriAd) to CAS.

\section{ACKNOWLEDGMENTS}

The authors thank Mrs. Cleusa Fumika Takakura for processing the paraffin embedded heart tissue for the electron microscopy analysis and PISA (Plataforma de Imagem na Sala de Autópsia), a core research facility at 
FMUSP, for performing the computed axial tomography on the heart of the corpse.

\section{REFERENCES}

1. Silva CA, Avcin T, Brunner HI. Taxonomy for systemic lupus erythematosus with onset before adulthood. Arthritis Care Res (Hoboken). 2012;64:1787-93.

2. Faco MM, Leone C, Campos LM, Febrônio MV, Marques HH, Silva CA. Risk factors associated with the death of patients hospitalized for juvenile systemic lupus erythematosus. Braz J Med Biol Res. 2007;40:993-1002.

3. Celes MR, Malvestio LM, Suadicani SO, Prado CM, Figueiredo MJ, Campos EC, et al. Disruption of calcium homeostasis in cardiomyocytes underlies cardiac structural and functional changes in severe sepsis. PLoS One. 2013;8:e68809.

4. Schellhammer F, Ansén S, Arnold G, Brochhagen HG, Lackner K. Myocardial calcification following septic shock. Cardiology. 2002;98:102-3

5. Sinicina I, Matevossian E, Fischer F, Mall G, Graw M. The petrified heart in sepsis. Virchows Arch. 2005;447:875-8.

6. Gladman DD, Ibañez D, Urowitz MB. Systemic lupus erythematosus disease activity index 2000. J Rheumatol. 2002;29:288-91.
7. Batista RS, Gomes AP, Dutra Gazineo JL, Balbino Miguel PS, Santana LA, Oliveira L, et al. Meningococcal disease, a clinical and epidemiological review. Asian Pac J Trop Med. 2017;10:1019-29.

8. Rossi MA, Santos CS. Sepsis-related microvascular myocardial damage with giant cell inflammation and calcification. Virchows Arch. 2003;443:87-92.

9. Auchet T, Regnier MA, Girerd N, Levy B. Outcome of patients with septic shock and high-dose vasopressor therapy. Ann Intensive Care. 2017;7:43.

10. Weisleder N, Taffet GE, Capetanaki Y. Bcl-2 overexpression corrects mitochondrial defects and ameliorates inherited desmin null cardiomyopathy. Proc Natl Acad Sci U S A. 2004;101:769-74.

11. Zheng G, Lyu J, Liu S, Huang J, Liu C, Xiang D, et al. Silencing of uncoupling protein 2 by small interfering RNA aggravates mitochondrial dysfunction in cardiomyocytes under septic conditions. Int J Mol Med. 2015;35:1525-36.

12. Soriano FG, Nogueira AC, Caldini EG, Lins MH, Teixeira AC, Cappi SB, et al. Potential role of poly(adenosine 5'-diphosphateribose) polymerase activation in the pathogenesis of myocardial contractile dysfunction associated with human septic shock. Crit Care Med. 2006;34:1073-9. 